\title{
The Influence of Node Velocity and Traffic Congestion on The Performance of AODV in MANET
}

\author{
Achmad Ubaidillah ${ }^{1}$, Miftachul Ulum, Heni Astutik \\ Department of Electrical Engineering \\ Trunojoyo University of Madura \\ Bangkalan, Indonesia \\ ${ }^{1}$ ca_achmed@yahoo.com
}

\author{
S. Ida Kholida \\ Department of Physics Education \\ Islamic university of Madura \\ Pamekasan, Indonesia
}

\begin{abstract}
There were many researches that discussed about performance of Ad hoc on Demand Distance Vector (AODV) in Mobile Ad-hoc Network (MANET). Some of them used mobile velocity and traffic congestion as variables in their observation. This research becomes very important because the writer of this paper was not sure of the result and the analysis of some previous research. So, this paper aims to explore about the impact of node velocity and traffic congestion to the performance and characteristic of AODV in MANET. The transport agents used are Transmission Control Protocol for File Transfer Protocol (TCP/FTP) and User Datagram Protocol for Constant Bit Rate (UDP/CBR). The research is applied by using Network Simulator 2 (NS-2). The performance is measured over some parameters, namely Overhead Routing (RO), Normalized Routing Load (NRL), and Packet Delivery Ratio (PDR). The contribution and the results obtained proved that the node velocity and traffic congestion can degrade the performance of AODV in MANET. It argued the other previous research claim that the node speed or velocity did not have influence to the performance of AODV MANET.
\end{abstract}

Keywords-Node velocity; traffic congestion; AODV; MANET

\section{INTRODUCTION}

The development of Computer network technology is very fast. Mobile network is the most popular and developed for next generation network. MANET is one among the answers. It is one of the wireless network technologies that consist a set of interconnected nodes to communicate each other. So, it works dynamically [1]. The network topology can change rapidly in accordance with existing conditions. Each node applies as a user and router simultaneously. So, routing protocol is very needed here. Router calculates and finds the shortest past of network. One of the most popular routing protocol is AODV.

There are many papers in routing protocol of MANET. [2] analized the characteristic of TCP and UDP on MANET with AODV routing protocol. Some performance parameters used are PDR, End-to-End Delay, Packet loss, RO, and Throughput. The research variable is pause time. [3] also analized the characteristic of TCP and CBR on MANET with different scenario, different parameters and different variables of the observations. even farther, there was a paper that proposed to enhance the routing protocol AODV on MANET. The performance parameters observed are End to End Delay, PDR, and Throughput. While the variable is amount of nodes [4]. The amount of node was used to apply the traffic conditions, namely light traffic, medium until congestion.

Comparison model was also used in the performance analysis of this topic. [5] and [6] compared the performance of TCP and UDP with different routing protocols such as AODV, DSDV and DSR on MANET. Even further, [7] compared the Performance of UDP/CBR and TCP/FTP on different Routing Protocols in VANET.

Some papers discussed about the influence of mobility speed on the network performance in MANET. Some of them investigated the influence of mobility speed on different routing protocol (AODV, DSDV and DSR) in MANET [8-10] discussed the influence of mobility speed on throughput in comparison of TCP/FTP and UDP/CBR in AODV MANET. It showed on graph of the research result that the increasing of mobility speed tends to have no effect on the throughput value. [11] also discussed the influence of maximum speed on throughput in comparison of TCP and CBR in AODV MANET. It showed on the research result that the increasing of maximum speed tends to degrade packet loss and average delay. The researcher of this paper was not satisfied with the result and the analysis of the other papers mentioned above. So, this paper aims to investigate the influence of mobile velocity and traffic congestion on the characteristic of AODV MANET. The transport agents used are TCP/FTP and UDP/CBR. The research is applied by using Network Simulator 2 (NS-2). The assessment uses some parameters, namely PDR, NRL, and RO. And the results will be compared with the other papers mentioned above.

\section{RESEARCH METODOLOGY}

In this paper, the simulation scenario is designed to investigate the influence of mobile velocity and traffic congestion on the performance of TCP/FTP and UDP/CBR network in MANET using NS-2. The routing protocol used here is AODV. In General, the system can be illustrated in Fig. 1 below. 


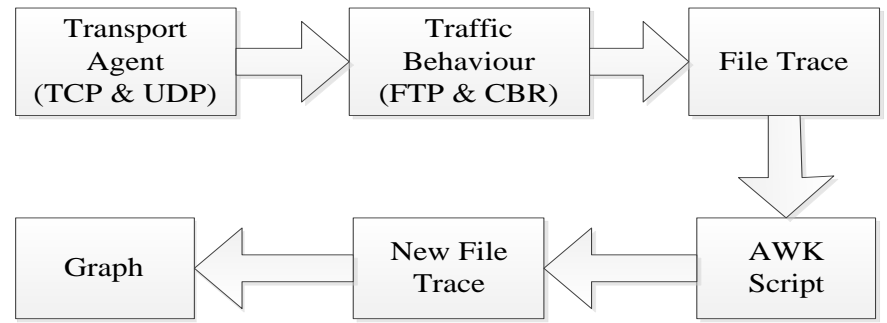

Fig. 1. Block diagram of the simulation with NS-2

Transport agent TCP is used for traffic behavior of FTP type, while UDP is used for traffic behavior of CBR type. The simulation output is a trace file. The values of RO, NRL and PDR can be calculated by using awk script. New trace file is produced from this process and it is applied to display graphs of the performance [12].

Routing Overhead (RO) is a total number of routing packets sent by a routing protocol in every delivery of data packet. This parameter is used to calculate the efficiency of the performance of the routing protocol at transmitter. The larger value of RO, the less network efficiency.

$$
R O=\frac{\sum \text { Routing Packets Sent }}{\sum \text { Data Packets Sent }}
$$

Normalized Routing Load (NRL) is number of routing packets transmitted on every data packet received at the destination. So, the value of this parameter is viewed from the terminal node. This parameter is used to calculate the efficiency of the performance of a routing protocol.

$$
N R L=\frac{\sum \text { Routing Packets Received }}{\sum \text { Data Packets Received }}
$$

Packet delivery ratio (PDR) is comparison of data packets sent to data packets received.

$$
P D R=\frac{\sum \text { Data Packets Received }}{\sum \text { Data Packets Sent }}
$$

Number of nodes in this simulation is varied from 10 nodes, 20 nodes until 30 nodes. Number of connection is also varied from 1 connection, 3 connections until 6 connections. This condition is applied to produce condition of light traffic until congested network. The nodes are set in motion. Each movement of nodes are set in random direction. The velocity of nodes is also varied from $5 \mathrm{~m} / \mathrm{s}, 10 \mathrm{~m} / \mathrm{s}$ until $20 \mathrm{~m} / \mathrm{s}$.

The simulation is applied in two scenario. In the first scenario, variation of nodes velocity is combined with variation of connection number. While in the second scenario, variation of node number is combined with variation of connection number. The simulation time is $60 \mathrm{~s}$, with area $\mathrm{X}=1000$ and $\mathrm{Y}=1000$.

\section{RESUlT AND DisCUSSION}

The values of RO, NRL and PDR from the first scenario of the simulation are shown in table 1 , table 2 and table 3 .

As shown in table 1 , the values of RO on UDP/CBR is smaller than TCP/FTP, either on 1 connections, 3 connections, and 6 connections. The average of RO are 0.003 for UDP/CBR and 0.086 for TCP/FTP. It happens because TCP is connection oriented protocol. TCP always rearranges data packets in each order specified. TCP applies acknowledgement, error detection and error correction mechanism, so it decreases the efficiency of transmission and enlarges the value of RO. Unlike TCP, UDP is connectionless protocol. Data is sent across the network in

\begin{tabular}{|c|c|c|c|}
\hline \multirow{2}{*}{ Velocity } & \multirow{2}{*}{ Connection } & \multicolumn{2}{|c|}{ RO } \\
\hline & & $T C P$ & $U D P$ \\
\hline \multirow{3}{*}{$5 \mathrm{~m} / \mathrm{s}$} & 1 & 0.014 & 0.001 \\
\hline & 3 & 0.061 & 0.003 \\
\hline & 6 & 0.091 & 0.002 \\
\hline \multirow{3}{*}{$10 \mathrm{~m} / \mathrm{s}$} & 1 & 0.048 & 0.004 \\
\hline & 3 & 0.068 & 0.004 \\
\hline & 6 & 0.112 & 0.004 \\
\hline \multirow{3}{*}{$20 \mathrm{~m} / \mathrm{s}$} & 1 & 0.111 & 0.004 \\
\hline & 3 & 0.139 & 0.003 \\
\hline & 6 & 0.170 & 0.003 \\
\hline & & 0.086 & 0.003 \\
\hline
\end{tabular}
chunks independently.

TABLE I. THE VALUES OF RO OF THE FIRST SCENARIO

The increasing of connection number from 1 connection, 3 connections until 6 connection in TCP/FTP causes the increasing of RO values sequentially $0.014,0.061$ and 0.091 for velocity $5 \mathrm{~m} / \mathrm{s}, 0.048,0.068$, and 0.112 for velocity $10 \mathrm{~m} / \mathrm{s}$, and $0.111,0.139$ and 0.170 for velocity $20 \mathrm{~m} / \mathrm{s}$. It means that the efficiency decreases. In TCP, the increasing of connection number means the increasing of routing packets, so it influences and decreases the efficiency at transmitter or RO. But it does not happen in UDP/CBR. The increasing of connection number does not influence the RO, because the data is transmitted in chunks independently [7].

The increasing of node velocity from $5 \mathrm{~m} / \mathrm{s}, 10 \mathrm{~m} / \mathrm{s}$ until 20 $\mathrm{m} / \mathrm{s}$ in TCP/FTP causes the increasing of RO values sequentially $0.014,0.048$ until 0.111 on 1 connection scenario, $0.061,0.068$ until 0.139 on 3 connections scenario, and $0.091,0112$ until 0.170 on 6 connections scenario. It influences and decreases the efficiency of transmision or RO because the velocity of nodes can change the routing calculation. It may produce more than one routing, so it can increase the value of RO. But it does not happen in UDP/CBR. The increasing of node velocity tends to have no influence on the RO, because the data is transmitted in chunks independently.

TABLE II. THE VALUES OF NRL OF THE FIRST SCENARIO

\begin{tabular}{|c|c|c|c|}
\hline \multirow{2}{*}{ Velocity } & \multirow{2}{*}{ Connection } & \multicolumn{2}{|c|}{ NRL } \\
\cline { 2 - 4 } & 1 & $\boldsymbol{T C P}$ & $\boldsymbol{U D P}$ \\
\hline \multirow{3}{*}{$5 \mathrm{~m} / \mathrm{s}$} & 3 & 0.024 & 0.006 \\
\cline { 2 - 4 } & 6 & 0.025 & 0.110 \\
\cline { 2 - 4 } & 1 & 0.160 & 0.123 \\
\hline \multirow{3}{*}{$10 \mathrm{~m} / \mathrm{s}$} & 3 & 0.111 & 0.034 \\
\cline { 2 - 4 } & 6 & 0.143 & 0.047 \\
\cline { 2 - 4 } & \multicolumn{2}{|c}{} \\
\hline
\end{tabular}




\begin{tabular}{|c|c|c|c|}
\hline \multirow{2}{*}{ Velocity } & \multirow{2}{*}{ Connection } & \multicolumn{2}{|c|}{ NRL } \\
\cline { 3 - 4 } & & $\boldsymbol{T C P}$ & $\boldsymbol{U D P}$ \\
\hline \multirow{3}{*}{$20 \mathrm{~m} / \mathrm{s}$} & 1 & 0.234 & 0.066 \\
\cline { 2 - 4 } & 3 & 0.182 & 0.026 \\
\cline { 2 - 4 } & 6 & 0.269 & 0.070 \\
\hline \multicolumn{2}{|c|}{ Average } & 0.164 & 0.066 \\
\hline
\end{tabular}

As shown in table 2, the average of NRL on TCP/FTP (0.164) is higher than UDP/CBR (0.066), because the transmitter efficiency (RO) of TCP/FTP is also higher than UDP/CBR. But the average of NRL, either on TCP/FTP and UDP/CBR are higher than the average of RO on TCP/FTP (0.086) and UDP/CBR (0.003), because not all transmitted data can be received in receiver. A part of them may be blocked because of traffic congestion or missed in channel.

In the same manner as $\mathrm{RO}$, the increasing of connection number from 1 connection, 3 connections until 6 connection in TCP/FTP causes the increasing of NRL values sequentially $0.024,0.025$ and 0.160 for velocity $5 \mathrm{~m} / \mathrm{s}, 0.111,0.143$, and 0.203 for velocity $10 \mathrm{~m} / \mathrm{s}$, and $0.234,0.182$ and 0.269 for velocity $20 \mathrm{~m} / \mathrm{s}$. It means that the efficiency of the receiver decreases. UDP/CBR also acts same characteristic with TCP/FTP. The NRL values on UDP/CBR also increases, sequentially $0.006,0.110$ and 0.123 for velocity $5 \mathrm{~m} / \mathrm{s}, 0.034$, 0.047 and 0.084 for velocity $10 \mathrm{~m} / \mathrm{s}$, and $0.066,0.026$ and 0.070 for velocity $20 \mathrm{~m} / \mathrm{s}$.

The increasing of node velocity from $5 \mathrm{~m} / \mathrm{s}, 10 \mathrm{~m} / \mathrm{s}$ until 20 $\mathrm{m} / \mathrm{s}$ in TCP/FTP causes the increasing of NRL values, sequentially $0.024,0.111$ until 0.234 on 1 connection scenario, $0.025,0.143$ until 0.182 on 3 connections scenario, and 0.160 , 0.203 until 0.269 on 6 connections scenario. The NRL values on UDP/CBR sequentially are $0.006,0.034$ until 0.066 on 1 connection scenario, $0.110,0.047$ until 0.026 on 3 connections scenario, and $0.123,0.084$ until 0.070 on 6 connections scenario. The faster node velocity is, the worse efficiency of NRL in TCP/FTP becomes. While on UDP/CBR, the node velocity tends to have no influence on the values of NRL.

The final parameter observed in the first scenario is PDR. The results are shown in table 3 . The average of PDR on TCP/FTP (96.517\%) is much higher than UDP/CBR (13.815\%), because TCP is connection oriented protocol, while UDP is connectionless protocol. With connection oriented type of TCP, there is guarantee that all data transmitted reach the receiver. While, with connectionless type UDP, there is no guarantee that all packets sent reach the receiver.

The increasing of connection number from 1 connection, 3 connections until 6 connections causes the increasing of traffic load, from light traffic until congested traffic. Even, the congested traffic causes the increasing of blocking probability. So, The increasing of connection number here produces the decreasing of PDR values on TCP/FTP and UDP/CBR.

TABLE III. THE VALUES OF PDR OF THE FIRST SCENARIO

\begin{tabular}{|c|c|c|c|}
\hline \multirow{2}{*}{ Velocity } & \multirow{2}{*}{ Connection } & \multicolumn{2}{|c|}{ PDR (\%) } \\
\cline { 2 - 4 } & 1 & $\boldsymbol{T C P}$ & $\boldsymbol{U D P}$ \\
\hline \multirow{3}{*}{$5 \mathrm{~m} / \mathrm{s}$} & 3 & 99.015 & 26.346 \\
\cline { 2 - 4 } & 6 & 97.017 & 4.248 \\
\cline { 2 - 4 } & 6 & 96.239 & 3.324 \\
\hline
\end{tabular}

\begin{tabular}{|c|c|c|c|}
\hline \multirow{2}{*}{ Velocity } & \multirow{2}{*}{ Connection } & \multicolumn{2}{|c|}{ PDR (\%) } \\
\cline { 2 - 4 } & & $\boldsymbol{T C P}$ & $\boldsymbol{U D P}$ \\
\hline \multirow{3}{*}{$10 \mathrm{~m} / \mathrm{s}$} & 1 & 98.00 & 25.493 \\
\cline { 2 - 4 } & 3 & 96.583 & 15.888 \\
\cline { 2 - 4 } & 6 & 95.651 & 9.213 \\
\hline \multirow{3}{*}{$20 \mathrm{~m} / \mathrm{s}$} & 1 & 96.707 & 11.838 \\
\cline { 2 - 4 } & 3 & 95.260 & 21.092 \\
\cline { 2 - 4 } & 6 & 94.180 & 6.895 \\
\hline \multicolumn{2}{|c|}{ Average } & 96.517 & 13.815 \\
\hline
\end{tabular}

In the same manner as RO and NRL, the increasing of nodes velocity from $5 \mathrm{~m} / \mathrm{s}, 10 \mathrm{~m} / \mathrm{s}$ until $20 \mathrm{~m} / \mathrm{s}$ gives negative effect and decreases the PDR values, either on TCP/FTP and UDP/CBR.

Comparing this result to another papers, [10] presented the relationship between speed of mobile node and throughput. It showed that the increasing of mobile node speed tends to have no influence on the performance of the network (throughput). This paper shows different result that the increasing of mobile node speed causes the decreasing of the performance of the network (value of PDR).

[11] also presented the relationship between maximum speed versus NRL, the relationship between maximum speed versus average delay and the relationship between maximum speed versus packet loss. The result of that paper showed that the relationship between maximum speed and NRL can not be concluded, because it doesn't tend to be up and doesn't tend to be down. The values of NRL are fluctuating. It also showed that the relationship between maximum speed and average delay also can not be concluded, because it doesn't tend to be up and doesn't tend to be down too. The values of the average delay are also fluctuating. In other side, it also showed that the increasing of maximum speed causes the packet loss tends to degrade. It means that the faster maximum speed is, the better performance obtained. In contradiction, this paper results that the faster node velocity is, the worse performance got.

In the second scenario, node number is varied from 10 nodes, 20 nodes until 30 nodes. This variation is combined with the variation of connection, from 1 connection, 3 connections until 6 connections. In the same manner as first scenario, the performance is defined by the vales of RO, NRL and PDR. The results are shown in table 4 for RO, table 5 for NRL and table 6 for PDR.

TABLE IV. THE VALUES OF RO OF THE SECOND SCENARIO

\begin{tabular}{|c|c|c|c|}
\hline \multirow{2}{*}{ Nodes } & \multirow{2}{*}{ Connection } & \multicolumn{2}{|c|}{ RO } \\
\hline & & $T C P$ & $U D P$ \\
\hline \multirow{3}{*}{10} & 1 & 0.068 & 0.003 \\
\hline & 3 & 0.096 & 0.020 \\
\hline & 6 & 0.084 & 0.005 \\
\hline \multirow{3}{*}{20} & 1 & 0.369 & 0.005 \\
\hline & 3 & 0.209 & 0.024 \\
\hline & 6 & 0.402 & 0.020 \\
\hline \multirow{3}{*}{30} & 1 & 0.245 & 0.027 \\
\hline & 3 & 0.655 & 0.081 \\
\hline & 6 & 1.183 & 0.093 \\
\hline \multicolumn{2}{|c|}{ Average } & 0.368 & 0.031 \\
\hline
\end{tabular}

From table 4, the increasing of node number and connection number tends to increase traffic load, either on TCP/FTP or 
UDP/CBR. The average of RO of 10 nodes are 0.082 for TCP/FTP and 0.009 for UDP/CBR. When the node number is 20 nodes, the average of RO becomes 0.327 for TCP/FTP and 0.016 for UDP/TCP. The average of RO becomes 0.694 for TCP/FTP and 0.067 for UDP/CBR when the node number is 30 nodes.

At congestion condition, there are many data packets that are blocked by network. It gives bigger impact on TCP/FTP than UDP/CBR, because TCP is a protocol that applies flow control. At congestion condition, TCP decreases the flow of data packets. It causes the data packets sent can be smaller than routing packets sent, so the $\mathrm{RO}$ value can be more than 1 . This condition can be seen at table 4 when node number is 30 nodes, connection number is 6 connections, then the $\mathrm{RO}$ value is 1.183 .

TABLE V. THE VALUES OF NRL OF THE SECOND SCENRIO

\begin{tabular}{|c|c|c|c|}
\hline \multirow{2}{*}{ Nodes } & \multirow{2}{*}{ Connection } & \multicolumn{2}{|c|}{ NRL } \\
\cline { 2 - 4 } & & $\boldsymbol{T C P}$ & $\boldsymbol{U D P}$ \\
\hline \multirow{3}{*}{10} & 1 & 0.149 & 0.012 \\
\cline { 2 - 4 } & 3 & 0.194 & 0.515 \\
\cline { 2 - 4 } & 6 & 0.178 & 0.146 \\
\hline \multirow{3}{*}{20} & 1 & 1.012 & 0.045 \\
\cline { 2 - 4 } & 3 & 0.622 & 0.378 \\
\cline { 2 - 4 } & 6 & 1.170 & 0.767 \\
\hline \multirow{3}{*}{30} & 1 & 1.084 & 0.317 \\
\cline { 2 - 4 } & 3 & 3.200 & 2.751 \\
\cline { 2 - 4 } & 6 & 1.477 & 10.156 \\
\hline \multicolumn{2}{|c}{} & & 1.676 \\
\hline
\end{tabular}

The average of NRL of the second scenario (table 5) is much higher than the average of RO (table 4). This scenario applied congested traffic. So, there are much data packets blocked by the network. It causes the average of NRL becomes higher than the average of RO. Even in the congestion condition, the NRL of UDP/CBR (10.156) is about 2 times higher than NRL of TCP/FTP (5.637). The average of NRL value on TCP/FTP (1.477) is also lower than UDP/CBR (1.676). This condition occurs because of flow control function of TCP that UDP does not have it. It causes the UDP data packets received become lower than the routing packets received.

TABLE VI. THE VALUES OF PDR OF THE SECOND SCENRIO

\begin{tabular}{|c|c|c|c|}
\hline \multirow{2}{*}{ Nodes } & \multirow{2}{*}{ Connection } & \multicolumn{2}{|c|}{ PDR (\%) } \\
\cline { 2 - 4 } & & $\boldsymbol{T C P}$ & $\boldsymbol{U D P}$ \\
\hline \multirow{3}{*}{10} & 1 & 95.340 & 42.115 \\
\cline { 2 - 4 } & 3 & 98.474 & 18.327 \\
\cline { 2 - 4 } & 6 & 96.023 & 7.560 \\
\hline \multirow{3}{*}{20} & 1 & 98.392 & 38.547 \\
\cline { 2 - 4 } & 3 & 97.448 & 17.659 \\
\cline { 2 - 4 } & 6 & 96.139 & 6.297 \\
\hline \multirow{3}{*}{30} & 1 & 98.761 & 33.499 \\
\cline { 2 - 4 } & 3 & 96.774 & 14.750 \\
\cline { 2 - 4 } & 6 & 95.146 & 4.424 \\
\hline \multicolumn{2}{|c}{} & 96.944 & 20.686 \\
\hline
\end{tabular}

Table 6 shows that the PDR values of the second scenario of TCP/FTP tend to be similar. The average of PDR value is about $96 \%$. With connection oriented and flow control system of TCP, congestion condition does not give impact to TCP/FTP system.
In UDP/CBR, the PDR values change according the congestion level. When the node number is 10 nodes, the PDR values of UDP decrease from $42.115 \%$ on 1 connection, $18.327 \%$ on 3 connections until $7.560 \%$ on 6 connections. When node number is 20 connections, it becomes lower than 10 nodes, namely $38.547 \%$ on 1 connection, $17.659 \%$ on 3 connections until $6.297 \%$ on 6 connections. And it becomes lower again when the node number is 30 nodes, namely $33.499 \%$ on 1 connection, $14.750 \%$ on 3 connections until $4.424 \%$ on 6 connections. Shortly, the increasing of traffic load in congestion causes the PDR value becomes to degrade.

\section{CONCUSIONS AND FURTHER RESEARCH}

This study has shown that the increasing of node velocity causes the value of RO and NRL on TCP/FTP tend to increse. But it tends to have no influence on the value of RO and NRL of UDP/CBR. In this case, the average of RO and NRL on TCP/FTP is much higher than the average of RO and NRL on UDP/CBR. In other side, it causes the PDR value tends to degrade either on TCP/FTP and UDP/CBR. This study contributed and proved that the increasing of node velocity can degrade the performance of AODV MANET and at the same time it argued another previous research claim that the node velocity did not have influence to the performance of AODV MANET.

In the second scenario, the increasing of traffic load also causes the value of RO tends to increase, either on TCP/FTP and UDP/CBR. The value of RO on TCP/FTP is higher than the value of RO on UDP/CBR. Even in congestion condition, the value of RO on TCP can be above 1 . So does the the value of NRL, the increasing of traffic load also causes the of NRL tends to increase, either on TCP/FTP and UDP/CBR. Even in congestion condition, the NRL of UDP/CBR can be higher than the NRL of TCP/FTP. In the other side, the increasing of traffic load causes the value of PDR on UDP/CBR tends to degrade. But it tends to have no influence on the PDR value of TCP/FTP. It means that the congestion condition tends to degrade only on the performance of UDP/CBR on AODV MANET. In this scenario, this study contributed to describe the performance behavior of AODV MANET in congestion condition in more detail.

Future research should explore the problems around the performance of routing protocol in MANET mathematically, in order to obtain more precise results. The enhancement of routing protocol performance is also highly recomended for future research.

\section{REFERENCES}

[1] F.H. Rahman, T.W. Au, W.S. Suhaili, and Y. Liu, "Performance of MANET with IPSec Under Jelly Fish Attack," Jt. Int. Inf. Technol., Mech. and Electron. Eng. Conf., Ser.. Adv. in Comp. Sci. Res., vol. 62, pp. 265268, 2017.

[2] Chaudharyand, M. Singh, and V. Singh, "Simulation and Study of AODV Routing Protocol Under CBR and TCP Traffic Source", Int. J. Future Comp. and Commun., vol. 3, no. 2, pp. 84-88, April 2014.

[3] A. Jain and R. Bharti, "Simulation and Performance Analysis of Throughput and Delay on Varying Time and Number of Nodes in MANET”, Int. J. Recent Res. and Rev., vol. VII, Issue 2, pp. 113-117, June 2014 . 
[4] A. Kaur and K. Singh, "Performance Analysis of AODV Protocol with TCP/FTP Traffic and Improved AODV Protocol with UDP/CBR Traffic for Optimizing Routing and QoS in MANET", Int. J. Adv. Res. in Comp. Sci., vol. 8, no. 3, pp. 596-601, March-April 2017.

[5] Kumar, Subodh, G.S. Agrawal, and S.K. Sharma, "Impact of CBR, FTP Traffic Patterns and Varying Node Density on Performance of Routing Protocols in MANETs," Int. J. Comp. Sci. and Mobile Comp., vol. 4 Issue 1, pp. 262-269, January 2015.

[6] B. Singh and R. Hans, "TCP and UDP Based Performance Analysis of AODV, DSR and DSDV Routing Protocols Under Different Traffic Conditions in Mobile Ad-Hoc Networks,” Int. J. Future Gener. Commun. and Netw., vol. 8, no. 2, pp. 73-92, 2015.

[7] Jaiswal, Deepika, and Dr. S.S. Bhadauria, "Performance Analysis of UDP/CBR \& TCP/FTP Traffic Under Reactive and Proactive Routing Protocols in VANET,” Int. Res. J. Eng. and Technol., vol. 2, pp. 700-709, July 2015.
[8] B.C. Singh, T.K. Godder, Md.S. Miah, and H.M.A. Awal, "Experimental Analysis of UDP Performance in Mobile Ad Hoc Networks with Different Routing Protocols and Varying Payload," Int. J. Distrib. and Parallel Syst., vol. 2, no. 6, pp. 107-113, November 2011.

[9] Md.A.R. Mahmud, Md.M. Rahman, and Md.S. Miah, "Performance Analysis of Routing Protocols for CBR Traffic in Mobile Ad-Hoc Networks," J. Inf., vol. 2, no. 1, pp. 1-9, 2016

[10] R. Sharma and K. Gupta, "Comparison Based Performance Analysis of UDP/CBR and TCP/FTP Traffic Under AODV Routing Protocol in MANET,” Int. J. Comp. Appl., vol. 56, no. 15, pp. 28-35, October 2012.

[11] S.K. Sharma and U. Barahdiya, "Performance Analysis of Different Traffic Sources TCP and CBR in AODV MANET,” Int. J. Eng. Res. and Mod. Educ., vol. 1, Issue 1, pp. 178-182, 2016.

[12] S.K. Poonam and A. Tomar, "A Comparative Analysis of Transport Layer Protocols,” Int. J. Inf. and Comp. Technol., vol. 4, no. 14, pp. 1403-1410, 2014. 